\title{
The correlation of lattice phonon frequencies with the superconducting transition temperatures of some (BEDT-TTF) salts
}

\author{
M. Dressel ${ }^{1}$ and J.E. Eldridge \\ Department of Physics, University of British Columbia, Vancouver, BC, Canada V6T 1Z1 \\ Jack M. Williams and H.H. Wang \\ Chemistry and Materials Science Divisions, Argonne National Laboratory, Argonne, IL 60439, USA
}

Received 16 June 1992

Revised manuscript received 9 September 1992

\begin{abstract}
The far-infrared powder absorption spectra, between $10 \mathrm{~cm}^{-1}$ and $350 \mathrm{~cm}^{-1}$, of seven BEDT-TTF salts that exhibit superconductivity at or near ambient pressure, have been measured as a function of temperature, between $5 \mathrm{~K}$ and $300 \mathrm{~K}$. The temperature dependences of the peak frequencies, along with the measured deuterium isotopic frequency shifts, separated the lattice from the internal modes. The frequencies of the observed lattice modes were found to increase with $T_{\mathrm{c}}$ in the three $\mathrm{k}$-phase salts in agreement with the reduced volume of the unit cell, and to have a mixed response in the three $\beta$-phase compounds. Furthermore, the general range and magnitude of the frequencies in the higher- $T_{\mathrm{c}} \mathrm{K}$-phase compounds were found to be larger than in the lower $T_{\mathrm{c}}$ $\beta$-phase compounds. Consequently, no evidence was found for a correlation between higher values of $T_{\mathrm{c}}$ and a softer lattice in these organic superconductors.
\end{abstract}

\section{Introduction}

In a recent article by Williams et al. [1] are listed the forty presently-known organic superconductors. These have all been discovered in just over the past decade, beginning with (TMTSF) ${ }_{2} \mathrm{PF}_{6}$ at $0.9 \mathrm{~K}$ under an applied pressure of $10 \mathrm{kbar}$ [2]. While the TMTSF donor molecule has produced seven superconductors, only (TMTSF) ${ }_{2} \mathrm{ClO}_{4}$ is an ambientpressure superconductor, with a $T_{\mathrm{c}}$ of $1.4 \mathrm{~K}$. The BEDT-TTF donor molecule, on the other hand, has been the source of twenty superconductors, most of them at ambient pressure, and among this family are those with the highest value of $T_{\mathrm{c}}$. These are $\mathrm{k}$ $(\mathrm{ET})_{2} \mathrm{Cu}(\mathrm{NCS})_{2}$ with $T_{\mathrm{c}}=10.4 \mathrm{~K} \mathrm{[3]}$ (ET is the common abbreviation for BEDT-TTF), $\kappa$ (ET) ${ }_{2} \mathrm{Cu}\left[\mathrm{N}(\mathrm{CN})_{2}\right] \mathrm{Br}$ with $T_{\mathrm{c}}=11.6 \mathrm{~K}[4,5]$, and K-(ET $)_{2} \mathrm{Cu}\left[\mathrm{N}(\mathrm{CN})_{2}\right] \mathrm{Cl}$ with $T_{\mathrm{c}}=12.8 \mathrm{~K}$ at $0.3 \mathrm{kbar}$

1 Present address: Department of Physics, University of California, Los Angeles, CA 90024-1547, USA.
[6]. There are several crystallographic phases adopted by the highly anisotropic and layered ET compounds, but the most common are the $\beta$-phase and the $\kappa$-phase. In the $\beta$-phase materials the two-dimensional layers of ET molecules, which are sandwiched between layers of anions, contain molecules whose planes are all parallel, whereas in the $k$-phase materials the molecules are arranged in dimer pairs with alternating orthogonal orientation. In the course of attempting to synthesize materials with higher $T_{\mathrm{c}} \mathrm{s}$ it was observed that there was a correlation between $T_{\mathrm{c}}$ and the unit-cell volume in the three isostructural $\beta$-phase superconductors $\beta^{*}$-(ET) ${ }_{2} I_{3}, \beta$-(ET) $)_{2} \mathrm{AuI}_{2}$, and $\beta-(E T)_{2} \mathrm{IBr}_{2}[7,8]$. More recently, Saito et al. [9] have included a few more ET superconductors, and have obtained the same correlation when $T_{\mathrm{c}}$ is plotted versus "effective volume", although the correction for the anion volume is not precise and the validity of this approach has not been proven. Fleming et al. [10] report a similar correlation for the cubic (isotropic) $\mathrm{M}_{3} \mathrm{C}_{60}$ family of superconductors. 
In the weak coupling BCS limit, one can write

$T_{\mathrm{c}} \sim \theta_{\mathrm{D}} \operatorname{cxp}\left(-\frac{1}{\lambda}\right)$,

where $\theta_{\mathbf{D}}$ is the Debye temperature and $\lambda$ is the electron-phonon coupling constant, given by

$\lambda=\frac{n\left(E_{\mathrm{F}}\right)\left\langle I^{2}\right\rangle}{M\left\langle\omega^{2}\right\rangle}$,

where $n\left(E_{\mathrm{F}}\right)$ is the density of states at the Fermi level, $\left\langle I^{2}\right\rangle$ is an electron-phonon matrix element averaged over the Fermi surface, $M$ is the mass of the vibrating molecule and $\left\langle\omega^{2}\right\rangle$ is an average of the square of the phonon frequencies.

In the case of strong coupling, McMillan [11] found

$T_{\mathrm{c}} \sim \frac{\theta_{\mathrm{D}}}{1.45} \exp \left(-\frac{1.04(1+\lambda)}{\lambda-\mu^{*}(1+0.62 \lambda)}\right)$,

where

$\lambda=2 \int_{o}^{\infty} \mathrm{d} \omega \alpha^{2} F(\omega) / \omega$

and $\mu^{*}$ is the effective screened Coulomb interaction. In eq. (4), $F(\omega)$ is the phonon density of states and $\alpha^{2}$ is a weighting factor proportional to the electron density of states at the Fermi level. In a subsequent treatment by Allen and Dynes [12] they showed that much better agreement between the various BCS superconductors and the theory could be obtained if the prefactor in eq. (3) was replaced by other forms, which involved various weighted integrals over the phonon densities of state.

The usual explanation for the "volume effect" on $T_{\mathrm{c}}$, as discussed above, it that it is equivalent to negative pressure, which will increase the organic molecule interplanar distances, reducing both the transfer integral and electronic bandwidth, which in turn increases the density of states at the Fermi level, $n\left(E_{\mathrm{F}}\right)$, causing $T_{\mathrm{c}}$ to rise (see eqs. (1) and (2)). An alternative explanation for these materials was proposed by Whangbo et al. $[1,13,14]$ in which they postulated that the increase in $\lambda$ results from the decrease in $\left\langle\omega^{2}\right\rangle$ in eq. (2), rather than an increase in $n\left(E_{\mathrm{F}}\right)$. This decrease in $\left\langle\omega^{2}\right\rangle$ would result from a reduction in the intermolecular forces (softening) in an expanded lattice. Certainly the decrease of $T_{\mathrm{c}}$ with applied pressure of the simple, nearly-free-electron BCS superconducting metals arises primarily from the pressure dependence of the phonon frequencies, since their electronic structure varies only slightly under pressure [15]. On the other hand, the increase in electron-phonon coupling, $\lambda$, between a good $\mathrm{BCS}$ superconductor like $\mathrm{Nb}$ and a better one like $\mathrm{Nb}_{3} \mathrm{Sn}$ comes from an increase in $n\left(E_{\mathrm{F}}\right)\left\langle I^{2}\right\rangle$ rather than a decrease in $M\left\langle\omega^{2}\right\rangle[12]$. This is due in part to the opposing effects of the phonon frequencies in $\lambda$ and $\theta_{\mathrm{D}}$ in eq. (1).

We therefore decided to measure the frequencies of some of the lattice modes of the ET superconductors by infrared spectroscopy in an attempt to see whether the higher values of $T_{\mathrm{c}}$ are accompanied by a lattice softening. The low site-symmetry of the ET molecules makes all of the lattice modes infrared active, and mixes the rotational and translational character, so that one may not also require Raman data. We emphasize, however, that the acoustic modes are not accessible to us and we recognize their importance in mediating conventional superconductivity, but the measurement of their frequencies would require inelastic neutron scattering. The lattice mode frequencies and eigenvectors depend on the crystal structure, which includes the number of formula units per unit cell, $Z$. A strict comparison can only be made, therefore, between isostructural crystals of the same phase, and even within this group between only those with the same $Z$. We have, therefore, investigated three $\kappa$-phase crystals and three $\beta$-phase crystals. A more general comparison will then be made between the compounds of these two distinctly different phases.

We have restricted our attention in this paper to the low-frequency lattice modes which should contribute the most to $\theta_{\mathrm{D}}$ in eq. (1) and also to $\lambda$ in eq. (2). It is possible that some high-frequency internal modes may also couple to the superconducting pairs, because their activated infrared intensity shows that they couple strongly to the normal charge carriers. A recent study [16], however, of the isotope effect in two ambient-pressure $\kappa$-phase ET superconductors, when two ${ }^{13} \mathrm{C}$ atoms are substituted for ${ }^{12} \mathrm{C}$ in the central double bond of the ET molecule, shows that the high-frequency totally-symmetric intramolecular mode near $1500 \mathrm{~cm}^{-1}, \nu_{3}\left(\mathrm{a}_{\mathrm{g}}\right)$, which is the most 
strongly activated mode [17], plays very little part in pair mediation.

\section{Experimental}

We have found in the past that a better signal-tonoise ratio in the far-infrared, when working with these organic conductors, is obtained by measuring the powder absorption rather than the reflectivity from single crystals [18]. (The crystals were prepared at the Argonne National Laboratory.) This is because the free carriers resulting from the high conductivity tend to screen out the small vibrational features. The powder method then allows one to probe the low-conductivity polarization ( $E$ perpendicular to the highly conducting planes), which is not possible by reflectivity, since the large crystal faces are parallel to the ET planes and the crystals are relatively thin. Furthermore, one can work with large powder samples, compared with small single crystals, resulting in a more intense signal. Accordingly, between 10 and $20 \mathrm{mg}$ of material were ground in a Nujol mull (Aldrich \# 062977) for about $10 \mathrm{~min}$ and transferred to one side of a thin wedged TPX window [19].

The vibrational features sharpen as the temperature is decreased and we therefore mounted the samples in a Janis Supervaritemp Dewar, in which the cooling is achieved by cold vaporized helium gas. This eliminates the problem of thermal gradients associated with the sinking of a sample to a cold finger. The temperature was controlled to within $1 \mathrm{~K}$ by means of a PAR 152 temperature controller. The dewar windows were $0.75 \mathrm{in}$. diameter, 0.002 in. thick polypropylene. The dewar tail was positioned at the focus of one of the sample chambers of a Bruker IFS $113 \mathrm{~V}$ Fourier spectrometer, and the detector was an Infrared Labs doped-germanium composite bolometer operating at 4.2. K. Spectra were typically obtained at $1 \mathrm{~cm}^{-1}$ resolution with 800 averaged scans taking a total time of $40 \mathrm{~min}$. Background spectra were obtained from a clean TPX window in $10 \mathrm{~min}$. The data between $15 \mathrm{~cm}^{-1}$ and $50 \mathrm{~cm}^{-1}$ were obtained using a $50 \mu \mathrm{m}$ mylar beam splitter, while between $50 \mathrm{~cm}^{-1}$ and $350 \mathrm{~cm}^{-1}$ a $6 \mu \mathrm{m}$ mylar beam splitter was used.

\section{Results and discussion}

Figure 1 shows the uncalibrated powder absorption coefficient at five temperatures between approximately $20 \mathrm{~cm}^{-1}$ and $350 \mathrm{~cm}^{-1}$, for $\mathrm{K}$ (ET $)_{2} \mathrm{Cu}\left[\mathrm{N}(\mathrm{CN})_{2}\right] \mathrm{Br}$. Several vibrational features are evident, and they sharpen at lower temperatures. The frequency of the peak of these features at $20 \mathrm{~K}$ are listed in table 1 . (The data obtained at $5 \mathrm{~K}$ exhibited more noise than the others due to the appearance of small liquid helium droplets in the sample chamber, when operating near the boiling temperature). Figure 2 shows similar spectra for $\mathrm{K}-$ $(\mathrm{ET})_{2} \mathrm{Cu}\left[\mathrm{N}(\mathrm{CN})_{2}\right] \mathrm{Cl}$, in which the signal-to-noise ratio at the high-frequency end is improved over that in fig. 1. Both of these figures are used in order to point out the similarity between them at all temperatures. This means that the $\mathrm{Cl}$ salt has not undergone the reported [6] metal-insulator phase transition near $50 \mathrm{~K}$, but has instead remained metallic and is superconducting at $5 \mathrm{~K}$. The $0.3 \mathrm{kbar}$ pressure required to suppress the insulating transition [20] is provided by the frozen mineral oil surrounding the powder particles in the Nujol mull. If no pressure were present, a semiconducting band gap would be clearly evident in the low-temperature spectra, in the

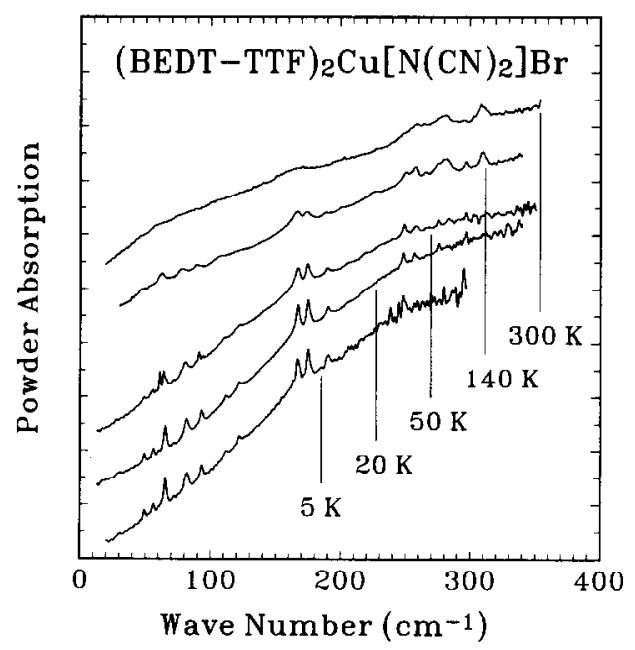

Fig. 1. The far-infrared powder absorption of $\kappa$ $(\mathrm{ET})_{2} \mathrm{Cu}\left[\mathrm{N}(\mathrm{CN})_{2}\right] \mathrm{Br}$ at five temperatures. The resolution is 1 $\mathrm{cm}^{-1}$, and the spectra, displaced for clarity, are the logarithmic ratios of the transmission through a clear window to that of the transmission through the powder and window. 
Table 1

The frequencies, in wavenumbers, of the powder absorption features at $20 \mathrm{~K}$, of three $\kappa$-phase (ET) ${ }_{2} \mathrm{X}$ salts ${ }^{\text {a) }}$

\begin{tabular}{|c|c|c|c|}
\hline $\begin{array}{l}\mathrm{X}= \\
T_{\mathrm{c}}=\end{array}$ & $\begin{array}{l}\mathrm{Cu}(\mathrm{NCS})_{2} \\
10.4 \mathrm{~K}\end{array}$ & $\begin{array}{l}\mathrm{Cu}\left[\mathrm{N}(\mathrm{CN})_{2}\right] \mathrm{Br} \\
11.6 \mathrm{~K}\end{array}$ & $\begin{array}{l}\mathrm{Cu}\left[\mathrm{N}(\mathrm{CN})_{2}\right] \mathrm{Cl} \\
12.8 \mathrm{~K} \text { at } 0.3 \mathrm{kbar}\end{array}$ \\
\hline \multicolumn{4}{|c|}{ Group } \\
\hline \multirow[t]{3}{*}{1.} & $62.9 \mathrm{w}$ & $48.9 \mathrm{w} \downarrow$ & $74.2 \mathrm{vw} \uparrow$ \\
\hline & $66.5 \mathrm{vw}$ & $55.9 \mathrm{w} \downarrow$ & $77.1 \mathrm{w} \uparrow$ \\
\hline & $69.4 \mathrm{vw}$ & $65.1 \mathrm{~s} \downarrow$ & \\
\hline \multirow[t]{2}{*}{2.} & $85.6 \mathrm{~m}$ & $81.2 \mathrm{~m} \downarrow$ & $86.3 \mathrm{~s} \uparrow$ \\
\hline & $91.6 \mathrm{~m}$ & $93.0 \mathrm{~m} \uparrow$ & $96.4 \mathrm{~m} \uparrow$ \\
\hline \multirow[t]{2}{*}{3.} & $102.9 \mathrm{w}$ & $111.6 \mathrm{w}$ & $112.8 \mathrm{w} \uparrow$ \\
\hline & & $121.7 \mathrm{w}$ & $126.8 \mathrm{~m} \uparrow$ \\
\hline \multirow[t]{3}{*}{4.} & $162.9 \mathrm{~s}$ & $167.0 \mathrm{~s} \uparrow$ & $168.7 \mathrm{~m} \uparrow$ \\
\hline & $166.1 \mathrm{~m}$ & $174.8 \mathrm{~s} \uparrow$ & $178.1 \mathrm{~s} \uparrow$ \\
\hline & $173.3 \mathrm{w}$ & $190.2 \mathrm{~m} \uparrow$ & $192.8 \mathrm{~m} \uparrow$ \\
\hline \multirow[t]{3}{*}{5.} & & $248.3 \mathrm{~m}$ & $247.6 \mathrm{~m}$ \\
\hline & & $256.7 \mathrm{w}$ & $257.7 \mathrm{w}$ \\
\hline & & $275.8 \mathrm{w}$ & $275.0 \mathrm{~m}$ \\
\hline \multicolumn{2}{|l|}{6.} & $297.0 \mathrm{w}$ & $296.5 \mathrm{~m}$ \\
\hline \multicolumn{2}{|l|}{7.} & & $310 \mathrm{br}$ \\
\hline
\end{tabular}

a) $\uparrow(\downarrow)$ indicates an increase (decrease) in lattice mode frequency compared with the column to its left. vw means very weak, w weak, $m$ medium, s strong and br broad.

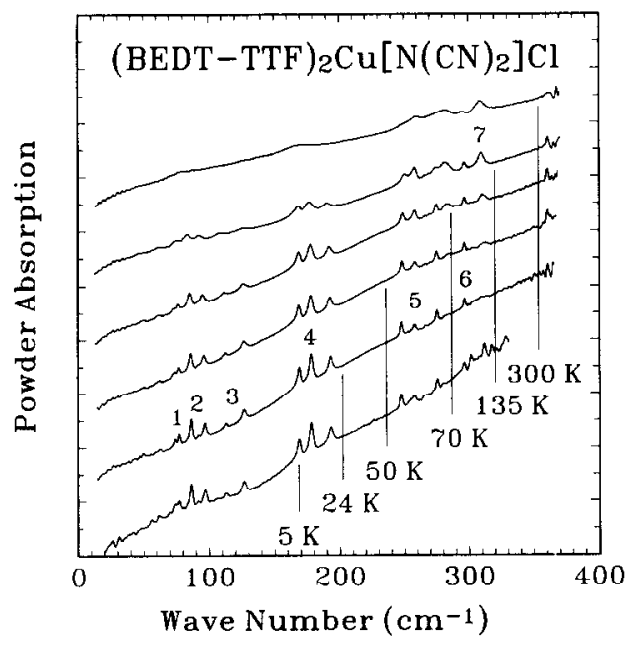

Fig. 2. The far-infrared powder absorption of $k$ (ET) ${ }_{2} \mathrm{Cu}\left[\mathrm{N}(\mathrm{CN})_{2}\right] \mathrm{Cl}$ at six temperatures. The frozen Nujol mull prevents the metal - semiconductor transition at $\sim 50 \mathrm{~K}$ in favor of a superconducting ground state. The resolution is $1 \mathrm{~cm}^{-1}$ and the spectra, displaced for clarity, are the logarithmic ratios of the transmission through a clear window to that of the transmission through the powder and window. form of reduced powder absorption below $1000 \mathrm{~cm}^{-1}$ [21]. The increasing slope of the spectra with lowered temperature is due to the higher conductivity in this region at low temperatures.

In order to separate the lattice modes from the internal modes, we plotted the frequencies of the features versus temperature, and also repeated the measurements on two of the compounds in which the eight hydrogen atoms of the ET molecule were replaced with deuterium. Figure 3 shows the temperature-dependence of the frequency of five stronger features in the Br-salt spectra of fig. 1 . The lower three of thesc show the usual softening of a few percent, as the temperature is raised, associated with lattice modes. The top two show a constant or increasing frequency, which is the signature of an internal mode. Confirmation of this comes from the isotope shifts. Figure 4 shows the spectra of the protonated $\left(h_{8}\right)$ and deuterated $\left(d_{8}\right) B r$ salt at $20 \mathrm{~K}$, with guide lines drawn between corresponding features in the two spectra. The frequencies of these features for this salt and the $\mathrm{Cu}(\mathrm{NCS})_{2}$ salt are listed in table 2 along with the isotopic ratios $\bar{\nu}_{\mathrm{h}} / \bar{\nu}_{\mathrm{d}}$. The features are arranged somewhat arbitrarily into groups which are labelled 1 through 7 on fig. 2, which shows the most extensive spectra. Some of the components in these groups 


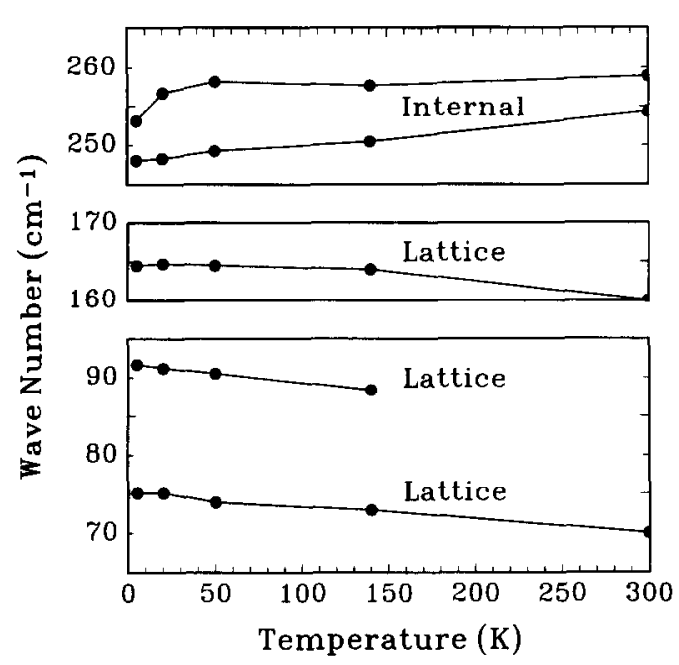

Fig. 3. The frequencies of the peaks of five of the larger features in the spectra of $(\mathrm{ET})_{2} \mathrm{Cu}\left[\mathrm{N}(\mathrm{CN})_{2}\right] \mathrm{Br}_{1}$, (fig. 1), as a function of temperature. This temperature dependence, along with the measured isotope shifts of table 2 and fig. 4 help to identify the lattice and internal modes.

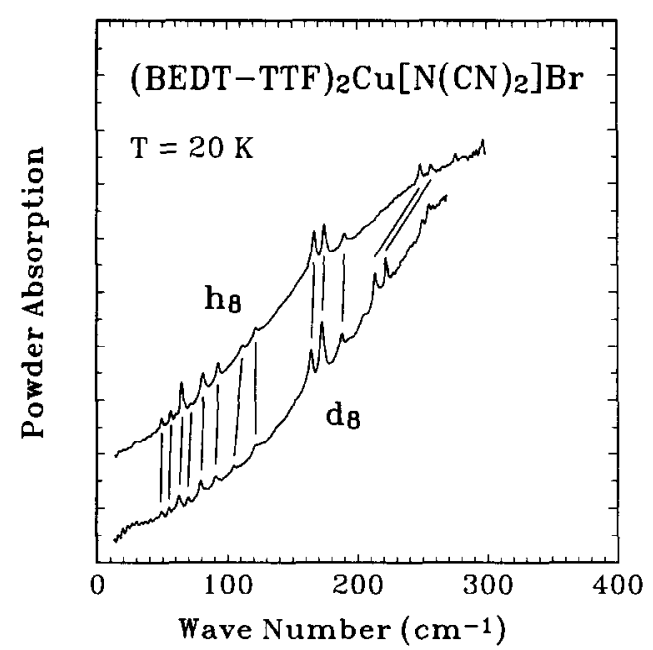

Fig. 4. The far-infrared powder absorption spectra of protonated and deuterated $\mathrm{K}-(\mathrm{ET})_{2} \mathrm{Cu}\left[\mathrm{N}(\mathrm{CN})_{2}\right] \mathrm{Br}$ at $20 \mathrm{~K}$. The lines correlate the features in the two spectra.

result from the large number of ET molecules per unit cell.

The expected isotopic ratios for translational and librational lattice modes, involving only the ET molecule, have been calculated and are listed in table 3 . They range from $1 \%$ for the translational and $R_{z}$ librations, to $3 \%$ for the other two librations. As al- ready mentioned, the lattice modes will be a mixture of these degrees of freedom. The molecular point group symmetry is approximately $D_{2 h}$ if the ET molecule is considered to be flat. The rotations will transform according to the symmetric $b_{g}$ irreducible representations, while the translations will transform according to the non-symmetric $b_{u}$ representations. The site symmetry of the ET molecule in the salts investigated here is general $\left(\mathrm{C}_{1}\right)$ which will mix the $b_{g}$ and $b_{u}$ modes. A centre of inversion at the ET site $\left(C_{i}\right)$ would be required to keep them separate. $K$ (ET) ${ }_{2} \mathrm{Cu}(\mathrm{NCS})_{2}$ has a monoclinic space group $P_{2_{1}}\left(C_{2}^{2}\right)$ with $Z=2$ and one layer per unit cell; $\kappa$ (ET) ${ }_{2}\left[\mathrm{~N}(\mathrm{CN})_{2}\right] \mathrm{X}, \mathrm{X}=\mathrm{Br}, \mathrm{Cl}$, are orthorhombic with space group $P_{\text {mna }}\left(D_{2 h}^{16}\right)$ and $Z=4$ and there are two layers per unit cell; $\beta$ - $(E T)_{2} X$ salts are triclinic with space group $P \overline{1}\left(C_{i}^{l}\right), Z=1$ and one layer per unit cell. In table 2 , therefore, the features in the first four groups are seen to have isotope ratios in the calculated lattice-mode range, with only one or two exceptions, and because of this, and the frequency temperature dependence, they are assigned to mixed lattice modes.

Group 5, in tables 1 and 2 and fig. 2 with a $16 \%$ isotope ratio, and the frequency temperature dependence shown in fig. 3 is clearly an internal mode, and is assigned to an out-of-plane $b_{3 u}$ mode, since there are no in-plane modes with this frequency and isotope shift in the results of the normal-coordinate calculations of Kozlov et al. [22]. We did not obtain an isotope shift for the feature labelled \#6 and so are unable to assign it. The \# 7 feature in table 1 and fig. 2 however, which appears only at high temperatures at $310 \mathrm{~cm}^{-1}$, has been seen by us before [17] and is the totally-symmetric internal mode $\nu_{11}\left(\mathrm{a}_{\mathrm{g}}\right)$. It is vibronically-activated and is therefore polarized in the highly-conducting plane. The optical activity is a result of charge oscillating between ET molecule dimers. Its appearance at high temperatures follows from the decrease of the underlying electronic conductivity in the conducting plane, which masks vibrational features at low temperatures.

Returning to table 1, which lists the frequencies of the vibrational features for the three $\kappa$-phase salts, we have indicated with an arrow whether the lattice mode frequencies (the first 4 groups) have increased or decreased with respect to those of the compound 
Table 2

The isotopic frequency ratios at $20 \mathrm{~K}$ of two $\mathrm{k}$-phase $(\mathrm{ET})_{2} \mathrm{X}$ salts

\begin{tabular}{|c|c|c|c|c|c|c|}
\hline \multirow[t]{2}{*}{$\mathbf{X}$} & \multicolumn{3}{|c|}{$\mathrm{Cu}(\mathrm{NCS})_{2}^{-}$} & \multicolumn{3}{|c|}{$\mathrm{Cu}\left[\mathrm{N}(\mathrm{CN})_{2}\right] \mathrm{Br}^{-}$} \\
\hline & $\mathbf{h}_{8}$ & $d_{8}$ & $\overline{\nu_{\mathrm{b}}} / \overline{\nu_{\mathrm{d}}}$ & $\mathrm{h}_{8}$ & $d_{8}$ & $\overline{\nu_{\mathrm{h}}} / \bar{\nu}_{\mathrm{d}}$ \\
\hline \multicolumn{7}{|c|}{ Group } \\
\hline \multirow[t]{3}{*}{1.} & 62.9 & 62.4 & 1.008 & 48.9 & 49.4 & 0.990 \\
\hline & 66.5 & - & - & 55.9 & 55.0 & 1.016 \\
\hline & 69.4 & 68.7 & 1.010 & 65.1 & 62.7 & 1.038 \\
\hline \multirow[t]{2}{*}{2.} & 85.6 & 83.4 & 1.026 & 81.2 & 79.3 & 1.024 \\
\hline & 91.6 & 93.8 & 0.098 & 93.0 & 90.9 & 1.023 \\
\hline \multirow[t]{2}{*}{3.} & 102.9 & 102.9 & 1.000 & 111.6 & 105.1 & 1.062 \\
\hline & - & - & - & 121.7 & 122.0 & 0.997 \\
\hline \multirow[t]{3}{*}{4.} & 162.9 & - & - & 167.0 & 164.6 & 1.015 \\
\hline & 166.1 & 163.9 & 1.013 & 174.8 & 172.6 & 1.013 \\
\hline & 173.3 & 170.2 & 1.018 & 190.2 & 188.3 & 1.010 \\
\hline \multirow[t]{2}{*}{5.} & - & - & - & 248.3 & 213.1 & 1.165 \\
\hline & - & - & - & 256.7 & 222.3 & 1.155 \\
\hline
\end{tabular}

Table 3

Calculated isotope ratios of the lattice modes involving only ET molecules ${ }^{\text {) }}$

\begin{tabular}{ll}
\hline Type of mode & Calculated $\bar{\nu}_{\mathrm{h}} / \overline{\nu_{\mathrm{d}}}$ \\
\hline Translational & 1.010 \\
Libration $\boldsymbol{R}_{\boldsymbol{x}}$ & 1.029 \\
Libration $\boldsymbol{R}_{\boldsymbol{y}}$ & 1.031 \\
Libration $\boldsymbol{R}_{\mathrm{z}}$ & 1.010 \\
\hline
\end{tabular}

a) The $z$-axis is along the central $\mathrm{C}=\mathrm{C}$ bond. The $y$-axis is in the molecular plane, while the $x$-axis is normal to it.

to the left with the lower $T_{\mathrm{c}}$. For the $\mathrm{Br}^{-}$salt, compared with the $\mathrm{Cu}(\mathrm{NCS})_{2}^{-}$salt there is a mixture of increases and decreases, but because of the different crystal structure and $Z$, we feel the comparison is not valid. In a previous mid-infrared study [23] we found that the frequencies of the normally-active $b_{2 u}$ internal modes decreased slightly in the $\mathrm{Br}^{-}$salt due to the looser packing in the conducting $a-c$ plane, compared with the conducting $b-c$ plane of the $\mathrm{Cu}(\mathrm{NCS})_{2}^{-}$salt (see table 4). The spacing between the planes, however, is smaller for the $\mathrm{Br}^{-}$salt than for the $\mathrm{Cu}(\mathrm{NCS})_{\overline{2}}^{-}$salt and this will produce increases in some lattice frequencies. (The unit cell volume divided by $Z$ is actually smaller for the $\mathrm{Br}^{-}$ salt, as opposed to statements in ref. [23] (see table 4.))

For the $\mathrm{Cl}$ salt, compared with the isostructural $\mathrm{Br}$ salt, however, it is seen that all of the lattice mode frequencies increase. This follows naturally from the smaller unit cell volume, which would produce a stiffer lattice (see column 7 in table 4 for data measured at $127 \mathrm{~K}$ ). For this group of three $\kappa$-phase compounds, therefore, in which $T_{\mathrm{c}}$ rises just over $2 \mathrm{de}$ grees as the unit cell volume decreases it appears that neither the electronic density of states nor lattice softness is responsible for this rise in $T_{\mathrm{c}}$. It is possible that the $\theta_{\mathrm{D}}$ prefactor is having an effect, but the mechanism is probably far more complicated.

The $\beta$-phase crystals that we have investigated are $\beta-(\mathrm{ET})_{2} \mathrm{IBR}_{2}\left(T_{\mathrm{c}}=2.8 \mathrm{~K}\right), \beta-(\mathrm{ET})_{2} \mathrm{AuI}_{2}\left(T_{\mathrm{c}}=5 \mathrm{~K}\right)$ and $\beta$-(ET $)_{2} \mathbf{I}_{3}$. The frozen mineral oil has probably again provided a pressure of at least $0.5 \mathrm{kbar}$, so that we have obtained the powder spectrum of $\beta_{\mathrm{H}}$ (or $\left.\beta^{*}\right)$ (ET) ${ }_{2} \mathrm{I}_{3}$ with a $T_{\mathrm{c}}$ of approximately $8 \mathrm{~K}$. As a possible check on this we also measured the spectrum of a sample of $\alpha_{\mathrm{t}}$-(ET) $)_{2} \mathrm{I}_{3}$, where $\alpha_{\mathrm{t}}$ is the transformed $\alpha$-phase with a $T_{\mathrm{c}}$ also near 7-8 K (see next paragraph). This transformation is accomplished by heating at $70^{\circ} \mathrm{C}$ for at least $70 \mathrm{~h}$. Figure 5 shows the temperature dependence of the spectrum of $\beta$ (ET) ${ }_{2} \mathrm{AuI}_{2}$ and the features in the $20 \mathrm{~K}$ spectrum have again been labelled in groups. Figure 6 shows the $20 \mathrm{~K}$ spectra of all of the compounds investigated in this study. These are arranged in order of increasing $T_{\mathrm{c}}$ proceeding from the bottom to the top of the figure, with the three $\mathrm{k}$-phase compounds on 
Table 4

Unit cell parameters and the superconducting transition temperatures of the $(\mathrm{ET})_{2} \mathrm{X}$ salts investigated in this study

\begin{tabular}{|c|c|c|c|c|c|c|c|c|c|}
\hline Salt & $T_{\mathrm{c}}(\mathrm{K})$ & $T_{\text {meas }}(\mathrm{K})$ & $a(\AA)$ & $b(\AA)$ & $c(\AA)$ & $\begin{array}{l}V\left(\hat{\mathrm{A}}^{3}\right) \\
\text { /charge }\end{array}$ & $V_{\text {eff }}\left(\AA^{3}\right)^{a)}$ & $Z$ & Ref. \\
\hline$\beta-(\mathrm{ET})_{2} \mathrm{IBr}_{2}$ & 2.7 & 120 & 6.589 & 8.820 & 14.988 & 807.1 & 692 & 1 & b) \\
\hline$\beta-(E T)_{2} \mathrm{AuI}_{2}$ & 5 & 120 & 6.570 & 8.891 & 15.299 & 821.7 & 707 & 1 & c) \\
\hline$\beta-(E T)_{2} \mathbf{I}_{3}$ & $8.1\left(\beta^{*}\right)$ & 120 & 6.561 & 9.013 & 15.173 & 829.2 & 704 & 1 & b) \\
\hline $\mathrm{K}-(\mathrm{ET})_{2} \mathrm{Cu}(\mathrm{NCS})_{2}$ & 10.4 & 104 & 16.382 & 8.402 & 12.833 & 822.6 & 714 & 2 & d) \\
\hline $\mathrm{K}-(\mathrm{ET})_{2} \mathrm{Cu}\left[\mathrm{N}(\mathrm{CN})_{2}\right] \mathrm{Br}$ & 11.8 & 127 & 12.878 & 29.681 & 8.484 & 810.7 & 720 & 4 & e) \\
\hline $\mathrm{K}-(\mathrm{ET})_{2} \mathrm{Cu}\left[\mathrm{N}(\mathrm{CN})_{2}\right] \mathrm{Cl}$ & 12.8 & 127 & 12.909 & 29.658 & 8.418 & 805.7 & 718 & 4 & f) \\
\hline
\end{tabular}

a) H. Yamochi, private communication.

b) P.C.W. Leung, T.J. Emge, A.J. Schultz, M.A. Beno, K.D. Carlson, H.H. Wang, M.A. Firestone and J.M. Williams, Solid State Commun. 57 (1986) 93. For the $\mathrm{I}_{3}^{-}$salt, at $100 \pm 30 \mathrm{~K}$ only the $\beta$-phase has been measured (cell parameters for average structure). For the $\beta^{*}$-phase, one would have to correct for effects of pressure (compressibility).

c) U. Geiser, H.H. Wang, C.E. Hammond, M.A. Firestone, M.A. Beno, K.D. Carlson, L. Nuñez and J.M. Williams, Acta Crystallogr. C 43 (1987) 656.

d) H. Urayama, H. Yamochi, G. Saito, S. Sato, A. Kawamoto, J. Tanaka, T. Mori, Y. Maruyama and H. Inokuchi, Chem. Lett. (1988) 463. The numbers given by Y. Watanabe, T. Sasaki, H. Sato and N. Toyota, J. Phys. Soc. Jpn. 60 (1991) 927 at $20 \mathrm{~K}$ are for a transformed cell (for comparison with the dicyanamide $\left[\mathrm{N}(\mathrm{CN})_{2}\right]$ salts) and represent otherwise unpublished results.

e) Ref. [4].

f) Ref. [6].

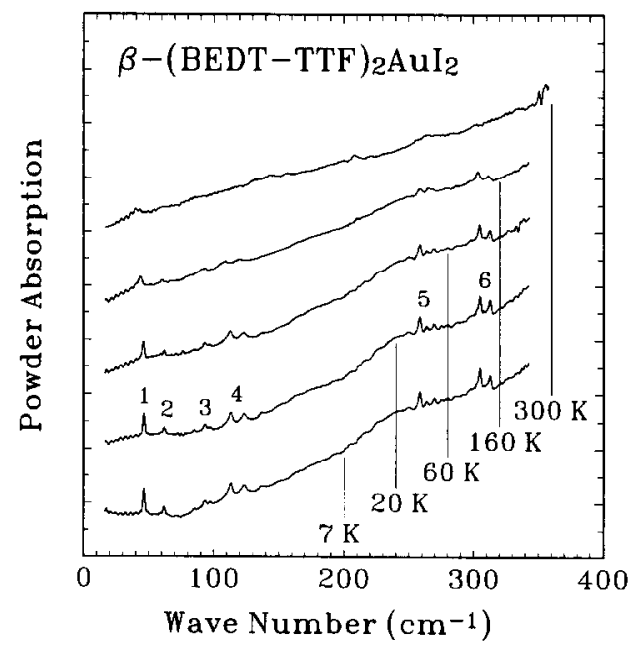

Fig. 5. The far-infrared powder absorption of $\beta-(E T)_{2} \mathrm{AuI}_{2}$ at five temperatures. The resolution is $1 \mathrm{~cm}^{-1}$ and the spectra, displaced for clarity, are the logarithmic ratios of the transmission through a clear window to that of the transmission through the powder and window.

top $(a-c)$. Table 5 lists the frequencies of the features in the three $\beta$-phase and the $\alpha_{t}$-phase compounds.

A comparison of the spectra $d$ and $e$ in fig. 6 as well as the last two columns in table 5 shows that the

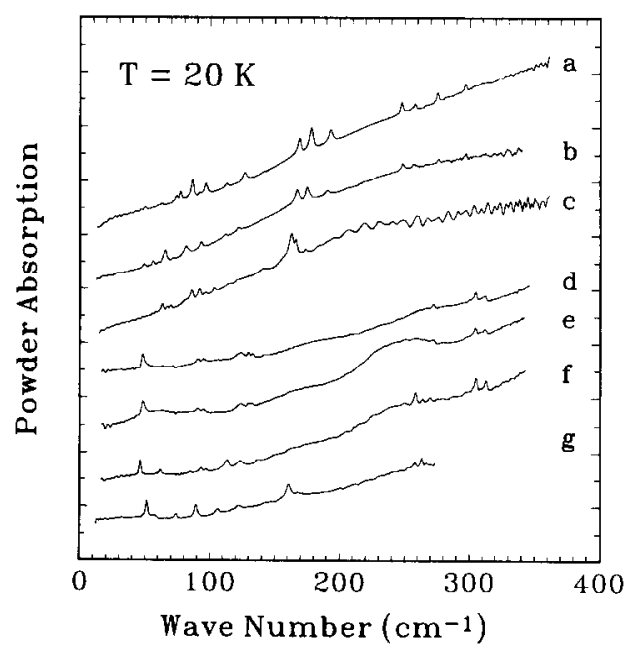

Fig. 6. The far-infrared powder absorption spectra of the seven compounds investigated in this study, displaced for clarity and arranged from bottom to top in order of increasing $T_{\mathrm{c}}$. They are (a) $\mathrm{k}-(\mathrm{ET})_{2} \mathrm{Cu}\left[\mathrm{N}(\mathrm{CN})_{2}\right] \mathrm{Cl}$, (b) $\mathrm{k}-(\mathrm{ET})_{2} \mathrm{Cu}\left[\mathrm{N}(\mathrm{CN})_{2}\right] \mathrm{Br}$, (c) $k$-(ET) ${ }_{2} \mathrm{Cu}(\mathrm{NCS})_{2}$, (d) $\beta^{*}$-(ET) $)_{2} \mathrm{I}_{3}$, (e) $\alpha_{4}$-(ET) $)_{2} \mathrm{I}_{3}$, (f) $\beta$ $(\mathrm{ET})_{2} \mathrm{AuI}_{2},(\mathrm{~g}) \beta-(\mathrm{ET})_{2} \mathrm{IBr}_{2}$. (See tables 1 and 5 for the values of $T_{\mathrm{c}}$ )

spectra of $\beta^{*}$-(ET) $)_{2} I_{3}$ and the $\alpha_{4}-(E T)_{2} I_{3}$ are virtually identical. Along with similar values of $T_{\mathrm{c}}$ and many other physical properties, this provides a further argument that the compounds may indeed be 
Table 5

The frequencies, in wavenumbers, of the powder absorption features, at $20 \mathrm{~K}$, of four $\beta$ or $\alpha_{t}$ phase (ET) ${ }_{2} \mathrm{X}$ salts ${ }^{\mathrm{a}}$

\begin{tabular}{|c|c|c|c|c|}
\hline $\begin{array}{l}\mathrm{X}= \\
T_{\mathrm{c}}=\end{array}$ & $\begin{array}{l}\beta-\mathrm{IBr}_{2} \\
2.8 \mathrm{~K}\end{array}$ & $\begin{array}{l}\beta-\mathrm{AuI}_{2} \\
5 \mathrm{~K}\end{array}$ & $\begin{array}{l}\beta^{*}-I_{3} \\
8 \mathrm{~K} \text { at } 0.5 \mathrm{kbar}\end{array}$ & $\begin{array}{l}a_{1}-I_{3} \\
7-8 \mathrm{~K}\end{array}$ \\
\hline 1. & $51.3 \mathrm{~s}$ & $46.3 \mathrm{~s} \downarrow$ & $48.2 \mathrm{~s} \uparrow$ & $48.4 \mathrm{~s}$ \\
\hline 2. & $73.5 \mathrm{w}$ & $75.0 \mathrm{vw} \uparrow$ & $71.3 \mathrm{vw} \downarrow$ & $74.0 \mathrm{vw}$ \\
\hline 3. & $\begin{array}{r}89.0 \\
-\quad\end{array}$ & $\begin{array}{l}92.8 \mathrm{w} \uparrow \\
96.9 \mathrm{w}\end{array}$ & $\begin{array}{l}90.6 \mathrm{w} \downarrow \\
947 \mathrm{w}\end{array}$ & $\begin{array}{l}90.6 \mathrm{w} \\
95.2 \mathrm{w}\end{array}$ \\
\hline \multirow[t]{4}{*}{4.} & $105.3 \mathrm{~m}$ & $113.3 \mathrm{~m} \uparrow$ & $123.9 \mathrm{~m} \uparrow$ & $124.1 \mathrm{~m}$ \\
\hline & $122.0 \mathrm{~m}$ & $123.4 \mathrm{~m} \uparrow$ & $129.7 \mathrm{w} \uparrow$ & $129.9 w$ \\
\hline & - & $137.2 \mathrm{w}$ & $132.6 \mathrm{vw} \downarrow$ & $132.8 \mathrm{vw}$ \\
\hline & $160.8 \mathrm{~s}$ & - & - & - \\
\hline \multirow[t]{2}{*}{ s. } & $257.7 w$ & $258.2 \mathrm{~s}$ & - & - \\
\hline & $263.0 \mathrm{~m}$ & $269.5 \mathrm{w}$ & $272.1 \mathrm{w}$ & $271.9 w$ \\
\hline \multirow[t]{2}{*}{6.} & - & $304.7 \mathrm{~s}$ & $304.2 \mathrm{~s}$ & $304.2 \mathrm{~m}$ \\
\hline & - & $312.4 \mathrm{~s}$ & $311.2 \mathrm{~m}$ & $311.4 \mathrm{~m}$ \\
\hline
\end{tabular}

a) $\uparrow(\downarrow)$ indicates an increase (decrease) in frequency compared with the column to its left. vw means very weak, w weak, m medium and s strong.

identical, and we are convinced that the Nujol mull has produced the $\beta^{*}$-(ET) $)_{2} \mathrm{I}_{3}$.

A comparison of the frequencies of the lattice modes (groups 1-4 in table 5) of the $\beta$-phase compounds, however, reveals mixed results. The trend towards higher $T_{\mathrm{c}}$ is not accompanied by uniformly increasing frequencies as was the case for the two $\mathrm{k}$ phase compounds. The arrows in table 5 shows a general increase in going from the $\mathrm{IBr}_{2}^{-}$to the $\mathrm{AuI}_{2}^{-}$salt but a mixed response in going to the $\mathrm{I}_{3}^{-}$ derivative. This mixed response might be due to the different enthylene group configuration in the $\beta^{*}$ (ET) ${ }_{2} \mathrm{I}_{3}$ salt compared with that in the $\beta-(\mathrm{ET})_{2} \mathrm{IBr}_{2}$ and $\beta$-(ET) ${ }_{2} \mathrm{AuI}_{2}$, which are isostructural. Group 4 between $110 \mathrm{~cm}^{-1}$ and $130 \mathrm{~cm}^{-1}$ is the only group with consistently increasing frequencies as $T_{\mathrm{c}}$ increases. In so far as the frequencies of these few lattice modes are an indication of lattice stiffness, however, there appears to be little evidence for the rise of $T_{\mathrm{c}}$ in this group of three $\beta$-phase compounds being due to lattice softness, in agreement with the $\kappa$-phase compounds. It is still possible, however, that the volume effect first noted by Williams et al. [7,8] may be operating through the increased density of states.

Two unexplained features exist in spectrum $g$ in fig. 6 , that of $\beta$-(ET) ${ }_{2} \mathrm{IBr}_{2}$. The first is the high wavenumber of the lowest-frequency strong feature at 51.3 $\mathrm{cm}^{-1}$, when one would have expected it to be less than that of the spectrum above if a general trend were being followed. The second and more serious question is the appearance of the strong feature at $160.8 \mathrm{~cm}^{-1}$, which is not present in the other two $\beta$ phase spectra, but resembles instead the group 4 of the $\mathrm{K}$-phase compounds. This latter group has an isotope shift indicating a mixture of translation and/or $R_{z}$ libration. The reason that more features are seen in the $\kappa$-phase spectra than in those of the $\beta$-phase is the larger value of $Z$ (number of formula units per

Table 6

Frequencies of the normal modes of the triatomic linear anions in the $\beta$-phase ET salts ${ }^{\text {a) }}$. Frequencies are in $\mathrm{cm}^{-1}$

\begin{tabular}{llll}
\hline (Double degenerate) & $\nu_{1}$ (Raman) & $\begin{array}{l}\nu_{2} \text { (IR) } \\
\text { (Doubly degenerate) }\end{array}$ & $\nu_{3}$ (IR) \\
\hline $\mathrm{I}_{3}^{-}$(solution) & 114 & 52 & 145 \\
$\mathrm{AuI}_{2}^{-}$(solid) & 158 & 67,59 & 210 \\
$\mathrm{BrIBr}^{-}$(solid) & 256 & 124 & 256 \\
\hline
\end{tabular}

\footnotetext{
a) K. Nakamoto, Infrared and Raman Spectra of Inorganic and Coordination Compounds (Wiley, New York, 1986).
} 
Table 7

Values of Debye temperature, $\theta_{\mathfrak{D}}$, extracted from specific heat studies

\begin{tabular}{lll}
\hline Compound & $T_{\mathrm{c}}$ & $\theta_{\mathrm{D}}$ \\
\hline$\alpha-(\mathrm{ET})_{2}\left(\mathrm{NH}_{4}\right) \mathrm{Hg}(\mathrm{SCN})_{4}$ & $1.1 \mathrm{~K}$ & $230 \pm 10 \mathrm{~K}^{2)}$ \\
$\beta-(\mathrm{ET})_{2} \mathrm{I}_{3}$ & $1.4 \mathrm{~K}$ & $197 \pm 5 \mathrm{~K}^{\mathrm{b})}$ \\
$\mathrm{K}-(\mathrm{ET})_{2} \mathrm{Cu}(\mathrm{NCS})_{2}$ & $10.4 \mathrm{~K}$ & $215 \pm 10 \mathrm{~K}^{\mathrm{c}}$ \\
$\mathrm{K}-(\mathrm{ET})_{2} \mathrm{Cu}\left[\mathrm{N}(\mathrm{CN})_{2}\right] \mathrm{Br}$ & $11.8 \mathrm{~K}$ & $210 \pm 15 \mathrm{~K}^{\mathrm{d})}$ \\
\hline
\end{tabular}

a) B. Andraka, G.R. Stewart, K.D. Carlson, H.H. Wang, M.D. Vashon and J.M. Williams, Phys. Rev. B 42 (1990) 9963.

b) G.R. Stewart, J.O'Rourke, G.W. Crabtree, K.D. Carlson, H.H. Wang, J.M. Williams, F. Gross and K. Andres, Phys. Rev. B 33 (1986) 2046.

c) B. Andraka, J.S. Kim, G.R. Stewart, K.D. Carlson, H.H. Wang and J.M. Williams, Phys. Rev. B 40 (1989) 11345.

d) B. Andraka, C.S. Jee, J.S. Kim, G.R. Stewart, K.D. Carlson, H.H. Wang, A.V. Strieby Crouch, A.M. Kini and J.M. Williams, Solid State Commun. 79 (1991) 57.

unit cell; see table 4). Does the presence of the 160.8 $\mathrm{cm}^{-1}$ feature in spectrum $\mathrm{g}$ therefore indicate some disorder or modulation, which has increased the size of the unit cell? It is also possible that there is a small amount of $\alpha-(E T)_{2} \mathrm{IBr}_{2}$ mixed with the $\beta-(\mathrm{ET})_{2} \mathrm{IBr}_{2}$. (The feature is not due to the anion since none of the vibrational features of the three linear anions, as listed in table 6, appear to be present with any strength in these seven powder spectra.)

Before leaving fig. 6, however, another comparison may be made. While it is not possible to compare individual features in the spectra of the $\kappa$-phase and the $\beta$-phase compounds, since the lattice modes will be different, one can observe that the overall distribution of lattice mode frequencies for the $\kappa$-phase compounds is higher than that for the $\beta$-phase. In spectrum a for example, the range is from $70 \mathrm{~cm}^{-1}$ to $200 \mathrm{~cm}^{-1}$, while in spectrum $\mathrm{d}$ the features start at $50 \mathrm{~cm}^{-1}$ and extend to only $135 \mathrm{~cm}^{-1}$. Figure 6 shows this trend quite well. Again one is led to speculate whether a suitable integral over the phonon frequencies in the prefactor of eq. (1) as considered in ref. [12] may be contributing to the increase in $T_{c}$ between these compounds. It is known that inaccurate results are obtained if the Debye frequency by itself is used [12]. Furthermore, the variations of $\theta_{\mathrm{D}}$ between compounds of these two phases is too small to produce the observed differences in $T_{\mathrm{c}}$. Table 7 lists the values of $\theta_{\mathrm{D}}$ extracted from specific heat measurements on various ET compounds.

What appears more likely, however, is that the equations (1-4) are inappropriate for these materials. Recent muon-spin-relaxation measurements [24] by Le et al. indicate non-BCS-type s wave pair- ing, and instead anisotropic pairing with line nodes in the energy gap. This would agree with our null result when we tried to observe the energy gap optically [25]. On a plot of $T_{\mathrm{c}}$ versus Fermi temperature, the organic salts, heavy fermion materials, and high- $T_{\mathrm{c}}$ cuprates all sit on the same straight line, far apart from the BCS-type metals [26].

\section{Conclusion}

We have measured the frequencies of some of the infrared-active lattice vibrations at various temperatures of three $\kappa$-phase and three $\beta$-phase organic superconductors based on the BEDT-TTF donor molecule. We found a general increase in these frequencies as $T_{\mathrm{c}}$ increases in the $\mathrm{K}$-phase compounds, consistent with the decreasing volume of the unit cell. We found a mixture of increases and decreases in the frequencies of the $\beta$-phase compounds as $T_{\mathrm{c}}$ increases, consistent with the combination of an increasing volume of the unit cell together with a decrease in one of the lattice constants. In general, the observed frequencies of the higher- $T_{\mathrm{c}} \mathrm{k}$-phase compounds are higher than those of the $\beta$-phase materials, although a strict comparison is not possible. We find no evidence that higher values of $T_{\mathrm{c}}$ in these materials are achieved through a softer lattice. On the contrary, it is possible that the higher opticalphonon frequencies may be contributing to the increase in $T_{\mathrm{c}}$, but it is likely to be a much more complicated situation than that described by a simple BCS expression. 


\section{Acknowledgements}

The work at the University of British Columbia (UBC) was supported by Grant No. 5-85653 from the Natural Sciences and Engineering Research Council (NSERC) of Canada. The work at Argonne National Laboratory was supported by the Office of Basic Energy Sciences, Division of Materials Sciences, of the US Department of Energy, Contract No. W-31-109-ENG.-38. MD acknowledges the support of the Deutsche Forschungsgemeinschaft (DFG). We wish to acknowledge useful discussions with James Schirber (Sandia National Laboratories), and thank U. Geiser (Argonne) for data in table 4.

\section{References}

[1] J.M. Williams, A.J. Schultz, U. Geiser, K.D. Carlson, A.M. Kini, H.H. Wang, W.-K. Kwok, M.-H. Whangbo and J.E. Schirber, Science 252 (1991) 1501.

[2] D. Jerome, A. Mazaud, M. Ribault and K. Bechgaard, J. Phys. (Paris) Lett. 41 (1980) L195.

[3] H. Urayama, H. Yamochi, G. Saito, K. Nozawa, T. Sugano, M. Kinoshita, S. Sato, K. Oshima, A. Kawamoto and J. Tanaka, Chem. Lett. (1988) 55.

[4] A.M. Kini et al., Inorg. Chem. 29 (1990) 2555.

[5] J.E. Schirber, D.L. Overmyer, J.M. Williams, A.M. Kini and H.H. Wang, Physica C 170 (1990) 231.
[6] J.M. Williams et al., Inorg. Chem. 29 (1990) 3272.

[7] J.M. Williams et al., Physica 136 B (1986) 371.

[8] J.M. Williams et al., Physica 143 B (1986) 346.

[9] G. Saito, T. Komatsu, T. Nakamura and H. Yamochi, Mat. Res. Symp. Proc. 247 (1992) 483.

[ 10] R.M. Fleming et al., Nature (London) 352 (1991) 787.

[11] W.L. McMillan, Phys. Rev. 167 (1968) 331.

[12] P.B. Allen and R.C. Dynes, Phys. Rev. B 12 (1975) 905.

[13] U. Geiser et al., Physica C 174 (1991) 475.

[14] M.-H. Whangbo, J.J. Novoa, D. Jung, J.M. Williams, A.M. Kini, H.H. Wang, U. Geiser, M.A. Beno and K.D. Carlson, in: "Organic Superconductivity", eds. V.Z. Kresin and W.A. Little (Plenum, New York, 1990) p. 243.

[15] J.W. Garland and K.B. Bennemann, "Superconductivity in $\mathrm{d}$ and f band metals", ed. D. Douglas, AIP Conf. Proc. \# 4 (1972) 255 .

[16] K.D. Carlson et al., Inorg. Chem. 31 (1992) 3346.

[17] K. Kornelsen et al., Phys. Rev. B 44 (1991) 5235.

[18] J.E. Eldridge and C.C. Homes, Phys. Rev. B 43 (1991) 13971.

[19] TPX is the Mitsui and Co. Ltd. trademark for methylpentene polymer.

[20] U. Welp et al., Phys. Rev. Lett. 69 (1992) 840.

[21] K. Kornelsen, et al., Solid State Commun. 81 (1992) 343.

[22] M.E. Kozlov, K.I. Pokhodnia and A.A. Yurchenko, Spectrochimica Acta A 45 (1989) 437.

[23] J.E. Eldridge, K. Kornelsen, H.H. Wang, J.M. Williams, A.V. Strieby-Crouch and D.M. Watkins, Solid State Commun. 79 (1991) 583.

[24] L.P. Le et al., Phys. Rev. Lett. 68 (1992) 1923.

[25] K. Kornelsen, J.E. Eldridge, H.H. Wang and J.M. Williams, Solid State Commun. 76 (1990) 1009.

[26] Y.J. Uemura et al., Phys. Rev. Lett. 66 (1991) 2665. 